\title{
CURVATURE OF THE DETERMINANT BUNDLE AND THE KÄHLER FORM OVER THE MODULI OF PARABOLIC BUNDLES FOR A FAMILY OF POINTED CURVES*
}

\author{
INDRANIL BISWAS ${ }^{\dagger}$ AND N. RAGHAVENDRA ${ }^{\ddagger}$
}

1. Introduction. Let $X$ be a compact Riemann surface and let $r$ and $d$ be relatively prime integers, with $r \geq 1$. The moduli space $\mathcal{U}_{X}(r, d)$ of stable vector bundles over $X$, of rank $r$ and degree $d$, is a non-singular projective variety, and furthermore, there exists a universal vector bundle $U$ over $\mathcal{U}_{X}(r, d) \times X$. The determinant of the cohomology of this family is a line bundle $L$ over $\mathcal{U}_{X}(r, d)$. This determinant line bundle is known to be ample [13]. Hence $L$ admits a positive Hermitian metric. One can ask whether there is a natural positive Hermitian metric on $L$. The answer to this is provided by a theorem of Quillen [20]: using this theorem of Quillen it is not difficult to construct a natural positive Hermitian metric on this line bundle $L$. One can ask the same question for the moduli space of stable vector bundles when $r$ and $d$ are arbitrary, not necessarily coprime, and more generally, for the moduli space of parabolic stable bundles with fixed parabolic data. This last question was answered in [9].

In another direction, there is the question of describing the behavior of such metrics when the Riemann surface itself varies over a family. Namely, let $f: X_{T} \longrightarrow T$ be a holomorphic family of Riemann surfaces, i.e, $X_{T}$ and $T$ are connected complex manifolds, and $f$ is a proper holomorphic submersion whose fibers are connected Riemann surfaces. Then, there is an associated family

$$
F: M_{T} \longrightarrow T
$$

of moduli spaces of stable vector bundles of given rank and degree. For each parameter $t \in T$, the fiber $F^{-1}(t)$ is the moduli space of stable vector bundles of rank $r$ and degree $d$ over $X_{t}=f^{-1}(t)$. There is a universal adjoint bundle over $M_{T}$. In [6] one of the authors works with the determinant of this universal adjoint bundle, constructs a natural Hermitian metric on this determinant line bundle and computes its curvature.

The aim of this paper is to extend these results to the situation of families of pointed Riemann surfaces and moduli spaces parabolic bundles over them. Let $f$ : $X_{T} \longrightarrow T$ be a smooth family of Riemann surfaces. Fix disjoint sections $s_{1}, \ldots, s_{n}$ of $f$. So we now have a family of Riemann surfaces with $n$ marked points parametrized by $T$. Let

$$
F: M_{T}^{P} \longrightarrow T
$$

be the relative moduli space of parabolic stable vector bundles of rank $r$ and parabolic degree zero with given parabolic data at the marked points. Then, there exists a determinant line bundle over $M_{T}^{P}$, which will be called the parabolic determinant line

\footnotetext{
${ }^{*}$ Received July 30, 1997; accepted for publication April 28, 1998. The first author thanks the Mehta Research Institute for hospitality while the work was carried out. Thanks are due to the referee for his comments, which are included as "concluding remarks".

†School of Mathematics, Tata Institute of Fundamental Research, Homi Bhabha Road, Bombay 400 005, INDIA (indranil@math.tifr.res.in). in).

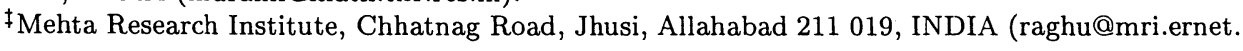


bundle [Section 4a]. We construct a natural Hermitian metric on this determinant bundle, and compute the curvature of the corresponding Hermitian connection [Section $4 \mathrm{e}]$.

The following is a brief description of the content of the paper. Let $X_{0}$ denote the complement of a finite subset of a compact connected Riemann surface $X$. In Section 2 we study a certain canonical affine bundle over the moduli space $M\left(X_{0}\right)$ of parabolic stable bundles over $X_{0}$ with fixed parabolic data over the parabolic points $X-X_{0}$. The affine bundle over $M\left(X_{0}\right)$ in question is the space of flat connections on $X_{0}$ satisfying a holonomy condition around the punctures, namely $X-X_{0}$. From this affine bundle a natural $(1,1)$-form on $M\left(X_{0}\right)$ is obtained. Section 3 is devoted to carrying out a similar study of a family of Riemann surfaces, and not just a fixed Riemann surface. In Section 4 we define the parabolic determinant line bundle for a family of marked Riemann surfaces. The curvature of the Hermitian connection corresponding to a natural Hermitian metric on this parabolic determinant line bundle has been expressed in terms of the above $(1,1)$-form.

2. Flat connections on a punctured Riemann surface. Let $X$ be a compact Riemann surface, or equivalently a connected smooth projective curve over $\mathbb{C}$. Take a finite subset

$$
D:=\left\{p_{1}, p_{2}, \ldots, p_{n}\right\} \subset X
$$

of $X$. The open Riemann surface $X-D$ will be denoted by $X_{0}$. The divisor $p_{1}+$ $p_{2}+\cdots+p_{n}$ on $X$ will also be denoted by $D$.

To any point $p_{i} \in D$, associate a conjugacy class $C_{i}$ in $U(r)$. Let $M\left(X_{0}\right)$ denote the space of equivalence classes of irreducible representations of the fundamental group $\pi_{1}\left(X_{0}\right)$ into the unitary group $U(r)$ such that the local holonomy, of the associated flat vector bundle over $X_{0}$, around any $p_{i} \in D$, i.e., the holonomy along a counterclockwise loop around $p_{i}$, is contained in the given conjugacy class $C_{i}$. Using results of [17] and [22], the space $M\left(X_{0}\right)$ gets identified with a moduli space of parabolic stable bundles over $X$ of parabolic degree zero. In particular, $M\left(X_{0}\right)$ has a natural structure of a complex manifold. The subset $D$ is the set of parabolic points, and the parabolic weights and their multiplicities at any $p_{i}$ are determined by the eigenvalues of an element of $C_{i}$ and their multiplicities, respectively.

The unique conjugacy class in $G L(r, \mathbb{C})$ which contains $C_{i}$ will be denoted by $\bar{C}_{i}$. Let $\bar{R}\left(X_{0}\right)$ denote the space of equivalence classes of representations of $\pi_{1}\left(X_{0}\right)$ into $G L(r, \mathbb{C})$ such that the local holonomy around any $p_{i} \in D$ is contained in the conjugacy class $\bar{C}_{i}$. The space $\bar{R}\left(X_{0}\right)$ has a natural structure of an affine algebraic variety over $\mathbb{C}$.

For any $\rho \in \bar{R}\left(X_{0}\right)$, we have a flat vector bundle of rank $r$ over $X_{0}$. This flat vector bundle has a natural extension, known as the Deligne extension, as a parabolic bundle over $X$ of parabolic degree zero. (See [15], [11] for the description of Deligne extension.) Let $V_{\rho}$ denote the parabolic bundle obtained from $\rho$ using Deligne extension.

Define

$$
R\left(X_{0}\right) \subseteq \bar{R}\left(X_{0}\right)
$$

to be the subspace consisting all $\rho \in \bar{R}\left(X_{0}\right)$ such that the corresponding parabolic bundle $V_{\rho}$ is actually parabolic stable. With this notation, it is known that $R\left(X_{0}\right)$ is a non-singular open subvariety of $\bar{R}\left(X_{0}\right)$. 
Let

$$
\pi: R\left(X_{0}\right) \longrightarrow M\left(X_{0}\right)
$$

denote the morphism which sends any $\rho$ to $V_{\rho}$. This map $\pi$ is holomorphic because of the following reason. For each $\rho_{0} \in R\left(X_{0}\right)$, there exists an analytic open neighborhood $U$ of $\rho_{0}$ in $R\left(X_{0}\right)$, and a holomorphic vector bundle, say $E$, over $X \times U$ with holomorphically varying flat connections, say $\nabla^{\rho}$, on $X_{0} \times \rho$, where $\rho \in U$, such that $\nabla^{\rho}$ is isomorphic to the flat connection corresponding to $\rho$. Moreover, the parabolic structures at $p_{i}$ for the individual $V_{\rho}$ combine together to give a natural parabolic structure on $E$ over the divisor $p_{i} \times U$. Now the holomorphicity of $\pi$ on $U$ is a consequence of a general property of the moduli space $M\left(X_{0}\right)$. The general property in question says that the classifying map for a holomorphic family of parabolic stable bundles is actually holomorphic.

For a parabolic stable bundle $V_{\rho}$, define $\operatorname{End}^{P}\left(V_{\rho}\right)$ to be the subsheaf of $\operatorname{End}\left(V_{\rho}\right)$ which preserves the parabolic flag at each parabolic point. Also, define $\operatorname{End}_{0}^{P}\left(V_{\rho}\right)$ to be the subsheaf of $\operatorname{End}^{P}\left(V_{\rho}\right)$ which is nilpotent with respect to the parabolic flag at each parabolic point. This means that if

$$
\left(V_{\rho}\right)_{p_{i}}=F^{1} \supset F^{2} \supset \ldots \supset F^{l} \supset F^{l+1}=0
$$

is the parabolic flag of the parabolic bundle $V_{\rho}$ at the parabolic point $p_{i}$, and $s$ is a local section of $\operatorname{End}_{0}^{P}\left(V_{\rho}\right)$, then

$$
s\left(F^{i}\right) \subseteq F^{i+1}
$$

Let $\Omega_{X}^{1}(\log D):=\Omega_{X}^{1} \otimes \mathcal{O}_{X}(D)$ be the sheaf of logarithmic differential forms.

The following proposition is well-known - see for example $\S 6$ of [10]:

Proposition 2.2. The holomorphic tangent space at $V_{\rho}$ to the moduli space $M\left(X_{0}\right)$, namely $T_{V_{\rho}} M\left(X_{0}\right)$, is naturally identified with $H^{1}\left(X, E n d^{P}\left(V_{\rho}\right)\right)$. The holomorphic cotangent space at $V_{\rho}$ is canonically isomorphic to

$$
H^{0}\left(X, \Omega_{X}^{1}(\log D) \bigotimes E n d_{0}^{P}\left(V_{\rho}\right)\right)
$$

For a vector bundle $E$ over $Y$, an affine bundle for $E$ - also called E-torsor - is a fiber bundle

$$
\gamma: Z \longrightarrow Y
$$

such that for each point $y \in Y$, the fiber $\gamma^{-1}(y)$ has an affine space structure for the vector space $E_{y}$, which depends holomorphically on $y$. In other words, if $s$ and $t$ are local holomorphic sections of $Z$ and $E$ respectively, then $s+t$ is also a holomorphic section of $Z$.

Lemma 2.3. The map $\pi: R\left(X_{0}\right) \longrightarrow M\left(X_{0}\right)$, defined in (2.1), is an affine bundle for the cotangent bundle $T^{*} M\left(X_{0}\right)$.

Proof. Take any $\rho \in R\left(X_{0}\right)$. The flat connection given by $\rho$ on the restriction of $V_{\rho}$ to $X_{0}$ extends as a logarithmic singular connection, which we shall denote by $\nabla^{\rho}$, on the vector bundle $V_{\rho}$, this being a general property of the Deligne extension. Recall that a logarithmic singular connection on $V_{\rho}$ is a homomorphism of sheaves

$$
\nabla^{\rho}: V_{\rho} \longrightarrow V_{\rho} \otimes \Omega_{X}^{1}(\log D)
$$


satisfying the Leibnitz identity. If $\nabla^{\prime}$ is another logarithmic singular connection on $V_{\rho}$ which is singular along $D$, then, by definition,

$$
\nabla^{\prime}-\nabla^{\rho} \in H^{0}\left(X, \operatorname{End}\left(V_{\rho}\right) \otimes \Omega_{X}^{1}(\log D)\right)
$$

For a logarithmic singular connection on $V_{\rho}$, defined as above, its residue is the endomorphism of the restriction of $V_{\rho}$ to the divisor $D$ given by the composition

$$
\left.V_{\rho} \longrightarrow V_{\rho} \otimes \Omega_{X}^{1}(\log D) \longrightarrow V_{\rho}\right|_{D}
$$

where the last homomorphism is defined using the natural projection (the residue map) of $\Omega_{X}^{1}(\log D)$ onto $\mathcal{O}_{D}$ (the kernel of this projection is $\Omega_{X}^{1}$ ). A logarithmic singular connection given by a Deligne extension has the further property that the imaginary parts of the eigenvalues of its residue are contained in the interval $(-1,0]$. The holonomy of any logarithmic connection around a puncture - along a counterclockwise loop - is the exponential of the residue at that puncture. Take any two logarithmic connections on the vector bundle $V_{\rho}$, say $\nabla$ and $\nabla^{\prime}$, given by Deligne extensions, such that they induce the same parabolic structure at all the points of $D$. In other words, at any point of $D$, the semi-simplification of the residue of $\nabla$ is equal to the semi-simplification of the residue of $\nabla^{\prime}$. From the above observations it is easy to deduce that

$$
\nabla^{\prime}-\nabla \in H^{0}\left(X, \Omega_{X}^{1}(\log D) \otimes \operatorname{End}_{0}^{P}\left(V_{\rho}\right)\right)
$$

On the other hand, for any $\theta \in H^{0}\left(X, \Omega_{X}^{1}(\log D) \otimes \operatorname{End}_{0}^{P}\left(V_{\rho}\right)\right)$, the operator $\nabla^{\rho}+\theta$ is a logarithmic singular connection on $V^{\rho}$ with the same local holonomy as $\nabla^{\rho}$ around the punctures. Furthermore, the eigenvalues of its residue are same as that of the residue of $\nabla^{\rho}$.

In view of the description of $T^{*} M\left(X_{0}\right)$ in Proposition 2.2, the proof of the lemma is now complete.

Let

$$
i: X_{0} \longrightarrow X
$$

denote the natural inclusion.

For any $\rho \in R\left(X_{0}\right)$, let $A d(\rho)$ denote the local system on $X_{0}$ associated to $\rho$ for the adjoint representation of $G L(r, \mathbb{C})$ into its Lie algebra $\mathfrak{g l}(r, \mathbb{C})$. The tangent space to $R\left(X_{0}\right)$ at $\rho$ has the following description.

Proposition 2.4. The holomorphic tangent space to $R\left(X_{0}\right)$ at any point $\rho$ is canonically isomorphic to $H^{1}\left(X, i_{*} A d(\rho)\right)$.

Proof. Let $H_{c}^{1}\left(X_{0}, A d(\rho)\right)$ denote the compactly supported first cohomology of the local system $A d(\rho)$. We know that $T_{\rho} R\left(X_{0}\right)$ is the image of $H_{c}^{1}\left(X_{0}, A d(\rho)\right)$ in $H^{1}\left(X_{0}, A d(\rho)\right)$ (Lemma 2.2 of [7], also Lemma 5.5 of [9]).

On the other hand, $H^{1}\left(X, i_{*} A d(\rho)\right)$ is realized as a subspace of $H^{1}\left(X_{0}, A d(\rho)\right)$ by the induced map of cohomology groups, induced by the inclusion $i$. It is an easy exercise to check that this subspace of $H^{1}\left(X_{0}, A d(\rho)\right)$ coincides with the image of $H_{c}^{1}\left(X_{0}, A d(\rho)\right)$ in $H^{1}\left(X_{0}, A d(\rho)\right)$ by the obvious map.

In Theorem 2.8 of [7] it was proved that the following pairing:

$$
H^{1}\left(X, i_{*} A d(\rho)\right) \bigotimes H^{1}\left(X, i_{*} A d(\rho)\right) \longrightarrow H^{2}\left(i_{*} A d(\rho) \otimes i_{*} A d(\rho)\right)
$$




$$
=H^{2}\left(X, i_{*}(A d(\rho) \otimes A d(\rho))\right) \stackrel{\text { trace }}{\longrightarrow} H^{2}\left(X, i_{*} \mathbb{C}\right)=H^{2}(X, \mathbb{C})=\mathbb{C}
$$

defines a symplectic structure on $R\left(X_{0}\right)$. The pairing $\operatorname{Ad}(\rho) \otimes \operatorname{Ad}(\rho) \longrightarrow \mathbb{C}$ is defined by taking point-wise trace, i.e., $A \otimes B \longmapsto \operatorname{trace}(A B)$. We shall denote this symplectic form on $R\left(X_{0}\right)$ by $\Theta$.

Recall that a closed submanifold $N$ of a symplectic manifold $M$ is said to be Lagrangian if for every point $p \in N, T_{p} N$ is a maximal isotropic subspace of $T_{p} M$.

PROPOSITION 2.6. The fibers of the map $\pi$ are Lagrangian submanifolds of $R\left(X_{0}\right)$ for the symplectic structure given by the form $\Theta$.

Proof. Let $\rho \in R\left(X_{0}\right)$, and let $F$ be the fiber of $\pi$ through $\rho$. The regular singular connection $\nabla=\nabla^{\rho}$ in $V_{\rho}$ induces a regular singular connection, also denoted $\nabla$, in $\operatorname{End}\left(V_{\rho}\right)$. Therefore we have the following short exact sequence of $\mathbb{C}_{X}$-modules on $X$, where $\mathbb{C}_{X}$ is the constant sheaf on $X$ with stalk $\mathbb{C}$ :

$$
0 \longrightarrow \operatorname{Ker}(\nabla) \longrightarrow \operatorname{End}\left(V_{\rho}\right) \stackrel{\nabla}{\longrightarrow} \Omega_{X}^{1}(\log D) \otimes \operatorname{End}\left(V_{\rho}\right) \longrightarrow 0 .
$$

Now consider the $\mathcal{O}_{X}$-submodule $\operatorname{End}^{P}\left(V_{\rho}\right)$ of $\operatorname{End}\left(V_{\rho}\right)$. The connection $\nabla$ does not preserve this submodule, but maps it onto $\Omega_{X}^{1}(\log D) \otimes \operatorname{End}_{0}^{P}\left(V_{\rho}\right)$ with kernel $i_{*} A d(\rho)$. Thus we have an induced short exact sequence of $\mathbb{C}_{X}$-modules

$$
0 \longrightarrow i_{*} A d(\rho) \longrightarrow \operatorname{End}^{P}\left(V_{\rho}\right) \stackrel{\nabla}{\longrightarrow} \Omega_{X}^{1}(\log D) \otimes \operatorname{End}_{0}^{P}\left(V_{\rho}\right) \longrightarrow 0 .
$$

Since $H^{0}\left(X, \operatorname{End}^{P}\left(V_{\rho}\right)\right) \cong \mathbb{C}$, the corresponding long exact sequence in cohomology simplifies to

$$
\begin{gathered}
0 \longrightarrow H^{0}\left(X, \Omega_{X}^{1}(\log D) \otimes \operatorname{End}_{0}^{P}\left(V_{\rho}\right)\right) \longrightarrow H^{1}\left(X, i_{*} \operatorname{Ad}(\rho)\right) \\
\longrightarrow H^{1}\left(X, \operatorname{End}^{P}\left(V_{\rho}\right)\right) \longrightarrow 0
\end{gathered}
$$

It is easy to see that the projection

$$
H^{1}\left(X, i_{*} A d(\rho)\right) \longrightarrow H^{1}\left(X, \operatorname{End}^{P}\left(V_{\rho}\right)\right)
$$

in (2.7) is precisely the differential, at $\rho$, of the map $\pi: R\left(X_{0}\right) \longrightarrow M\left(X_{0}\right)$. Therefore, the tangent space to the fiber $F$ at $\rho$, which equals the kernel of $\mathrm{d} \pi_{\rho}$ because $\pi$ is a submersion, is canonically isomorphic to $H^{0}\left(X, \Omega_{X}^{1}(\log D) \otimes \operatorname{End}_{0}^{P}\left(V_{\rho}\right)\right)$. From the definition of the symplectic form on $R\left(X_{0}\right)$ it is immediate that its restriction to $H^{0}\left(X, \Omega_{X}^{1}(\log D) \otimes \operatorname{End}_{0}^{P}\left(V_{\rho}\right)\right)$ is zero. Thus $T_{\rho} F$ is isotropic. Since $\operatorname{dim} R\left(X_{0}\right)=$ $2 \operatorname{dim} F$, it follows that $T_{\rho} F$ is maximal isotropic.

The projection $H^{1}\left(X, i_{*} A d(\rho)\right) \longrightarrow H^{1}\left(X, \operatorname{End}^{P}\left(V_{\rho}\right)\right)$ in $(2.7)$ is the differential of the map $\pi$ at $\rho$. Since $\pi^{-1}(\pi(\rho))$ is Lagrangian, there is an induced non-degenerate pairing between $H^{0}\left(X, \Omega_{X}^{1}(\log D) \otimes \operatorname{End}_{0}^{P}\left(V_{\rho}\right)\right)$ and $H^{1}\left(X, \operatorname{End}^{P}\left(V_{\rho}\right)\right)$, which is the obvious pairing between the tangent and the cotangent bundle of $M\left(X_{0}\right)$. Since the following isomorphism, which is easy to check, is valid:

$$
\mathcal{O}_{X}(D) \otimes \operatorname{End}_{0}^{P}\left(V_{\rho}\right)=\operatorname{End}^{P}\left(V_{\rho}\right)^{*}
$$

Serre duality is applicable.

Let

$$
\psi: M\left(X_{0}\right) \longrightarrow R\left(X_{0}\right)
$$


denote the obvious $C^{\infty}$ embedding induced by the inclusion of $U(r)$ in $G L(r, \mathbb{C})$. We want to calculate $\bar{\partial} \psi$ which measures how much $\psi$ differs from being a holomorphic embedding.

To define $\bar{\partial} \psi$ first note the following: let $A: V \rightarrow W$ be a $\mathbb{R}$-linear homomorphism between two complex vector spaces. The homomorphism $\tilde{A}: V \longrightarrow W$ defined by

$$
\tilde{A}(v):=\frac{A(v)+\sqrt{-1} A(\sqrt{-1} v)}{2}
$$

is conjugate linear, i.e., $\tilde{A}(\sqrt{-1} v)=-\sqrt{-1} A(v)$, and it vanishes if and only if $A$ is complex linear.

We apply the construction in (2.9) point-wise to the differential of $\psi$. Since the holomorphic tangent bundle is isomorphic - as a real vector bundle - to the real tangent bundle, we have a $\mathbb{R}$-linear homomorphism

$$
d \psi: T M\left(X_{0}\right) \longrightarrow \psi^{*} T R\left(X_{0}\right)
$$

Applying (2.9) to $d \psi$ we have a conjugate linear homomorphism $\widetilde{d \psi}$ from $T M\left(X_{0}\right)$ to $\psi^{*} T R\left(X_{0}\right)$ which vanishes if and only if $\psi$ is a holomorphic map.

For any $v \in T_{\nu} M\left(X_{0}\right)$, we have

$$
d \pi(\widetilde{d \psi}(v))=d \pi((d \psi(v)+\sqrt{-1} d \psi(\sqrt{-1} v)) / 2)=v+(\sqrt{-1})^{2} v=0
$$

The second equality is a consequence of the holomorphicity of the projection $\pi$. So $\widetilde{d \psi}$ is actually a conjugate linear homomorphism, namely

$$
\widetilde{d \psi}: T M\left(X_{0}\right) \longrightarrow \psi^{*}(\operatorname{kernel}(d \pi))=T^{*} M\left(X_{0}\right)
$$

The last equality was obtained in the proof of Proposition 2.6.

Equivalently, $\widetilde{d \psi}$ is a section of $T^{*} M\left(X_{0}\right) \otimes \overline{T^{*} M\left(X_{0}\right)}$. The following theorem says that this section is skew-hermitian and also identifies it as the pull-back of the symplectic form $\Theta$ on $R\left(X_{0}\right)$.

THEOREM 2.11. $\widetilde{d \psi}$ is skew-hermitian, i.e., it is a $(1,1)$-form on $M\left(X_{0}\right)$. Moreover this form coincides with $\psi^{*} \Theta / 2$.

Proof. We first note that for any unitary representation $\rho \in M\left(X_{0}\right)$, the Deligne extension of the flat vector bundle $A d(\rho)$ over $X_{0}$, that corresponds to the adjoint representation of $U(r)$ in $\mathfrak{g l}(r, \mathbb{C})$, is the vector bundle End ${ }^{P}\left(V_{\rho}\right)$. This observation is an easy consequence of the definition of the Deligne extension.

If we replace $V$ in the definition of $\widetilde{\Omega}_{X}^{1}(\mathcal{M})$, given in page 189 of [23], by the above local system $A d(\rho)$, then we get $\Omega_{X}^{1}(\log D) \otimes \operatorname{End}_{0}^{P}\left(V_{\rho}\right)$.

Now by Theorem D2(b), page 189, of [23] (or Theorem 5.1(b), page 163, of [24]) we have:

$$
H^{1}\left(X, i_{*} A d(\rho)\right)=H^{0}\left(X, \Omega_{X}^{1}(\log D) \otimes \operatorname{End}_{0}^{P}\left(V_{\rho}\right)\right) \bigoplus H^{1}\left(X, \operatorname{End}^{P}\left(V_{\rho}\right)\right)
$$

In other words, if $\rho$ in (2.7) is unitary, then the exact sequence (2.7) has a canonical splitting given by $(2.12)$.

The dual local system $A d(\rho)^{*}$ is isomorphic to $A d(\rho)$ since the isomorphism of $\mathfrak{g l}(r, \mathbb{C})$ with $\mathfrak{g l}(r, \mathbb{C})^{*}$, given by $A \otimes B \longmapsto \operatorname{trace}(A B)$, commutes with the adjoint 
action of $G L(r, \mathbb{C})$. Thus Theorem D2(c), page 189, of [23] (or Theorem 5.1(c), page 163 , of [24]) we have a conjugate linear isomorphism:

$$
j: H^{1}\left(X, \operatorname{End}^{P}\left(V_{\rho}\right)\right) \longrightarrow H^{0}\left(X, \Omega_{X}^{1}(\log D) \otimes \operatorname{End}_{0}^{P}\left(V_{\rho}\right)\right)
$$

Recall the descriptions of the tangent spaces of $M\left(X_{0}\right)$ and $R\left(X_{0}\right)$ given in Propositions 2.2 and 2.4. For the embedding $\psi$ it is easy enough to check that the differential $d \psi$ in $(2.10)$ is

$$
\omega \longmapsto \omega+j(\omega) \in H^{1}\left(X, i_{*} A d(\rho)\right)
$$

in the decomposition (2.12), where $\omega \in H^{1}\left(X, \operatorname{End}^{P}\left(V_{\rho}\right)\right)$. That the homomorphism defined in (2.14) is not $\mathbb{C}$-linear reflects the fact that the map $\psi$ is not holomorphic.

The observation that $d \psi$ coincides with the homomorphism given by (2.14) immediately implies that

$$
\widetilde{d \psi}(\omega)=j(\omega)
$$

Since the homomorphism $j$ corresponds to taking $\star$ of harmonic $L^{2}$ forms, we have

$$
j(\omega)\left(\omega_{1}\right)=-\overline{j\left(\omega_{1}\right)(\omega)}
$$

This equality together with $(2.15)$ implies that $\widetilde{d \psi}$ is skew-hermitian, i.e., it is a $(1,1)$-form on $M\left(X_{0}\right)$.

Now, in view of the fact that $d \psi$ coincides with the homomorphism in (2.14), the equality

$$
\widetilde{d \psi}=\psi^{*} \Theta / 2
$$

is a consequence of the definition of $\Theta$ given in (2.5).

For a vector bundle $E$ over $Y$, the space of all isomorphism classes of affine bundles for $E$ are parametrized by $H^{1}(Y, E)$. For an affine bundle $F$, any two local identifications with $E$ differ by a local section of $E$ which gives an one cocycle. Given a $C^{\infty}$ section $s$ of an affine bundle $F$, the section $\widetilde{d s}$ of $\Omega_{Y}^{0,1} \otimes E$, defined following the construction in (2.10), actually represents the element in $H^{1}(Y, E)$ that corresponds to $F$.

Thus, by Theorem 2.11 , the form $\psi^{*} \Theta / 2$ represents the cohomology class corresponding to the affine bundle for $\Omega_{M\left(X_{0}\right)}^{1}$, namely $R\left(X_{0}\right)$.

If $R\left(X_{0}\right)$ is complete then the symplectic $(1,1)$-form $\psi^{*} \Theta / 2$ represents a nonzero cohomology class. So, in that case, $R\left(X_{0}\right)$ over $M\left(X_{0}\right)$ is actually a nontrivial affine bundle for $\Omega_{M\left(X_{0}\right)}^{1}$.

3. Family of Riemann surfaces. Fix, as before, a parabolic data for $n$ parabolic points, which is same as fixing $r$ orbits, say $C_{1}, \ldots, C_{n}$, in $U(r)$ for the conjugation action.

Let

$$
f: X_{T} \longrightarrow T
$$

be a smooth family of Riemann surfaces. By this we mean that $T$ and $X_{T}$ are connected complex manifolds and $f$ is a proper smooth map of relative (complex) 
dimension one and with connected fibers. For $t \in T$, the Riemann surface $f^{-1}(t)$ will be denoted by $X_{t}$. Let

$$
s_{i}: T \longrightarrow X_{T}, \quad i=1,2, \ldots, n
$$

be $n$ disjoint holomorphic sections of $f$, i.e., $f \circ s_{i}=i d$, and the images of $s_{i}$ are all disjoint. So $T$ parametrizes a family of Riemann surfaces with $n$ marked points.

Let

$$
F: M_{T}^{P} \longrightarrow T
$$

be the relative moduli space of parabolic stable bundles of rank $r$ and parabolic degree zero, and with the given parabolic data on the marked points. In other words, for any $t \in T$, the space $M_{t}^{P}:=F^{-1}(t)$ is the moduli space of parabolic stable bundles over $X_{t}$ of rank $r$ and parabolic degree zero and with the given parabolic structure over the parabolic points $s_{i}(t), 1 \leq i \leq n$.

The space $M_{T}^{P}$ has the following universal property: for a holomorphic map $p$ : $S \longrightarrow T$, if

$$
E \rightarrow X_{T} \times_{T} S
$$

is a vector bundle over the fiber product with parabolic structures (of the given type) over all divisors image $\left(s_{i}\right) \times_{T} S$ such that for any $s \in S$ the parabolic bundle over $X_{p(s)}$, obtained by restricting $E$ to $X_{p(s)}$, is parabolic stable - then the obvious map

$$
\phi_{E}: S \longrightarrow M_{U}^{P}
$$

which assigns to any $s$ the point in $M_{p(s)}^{P}$ that corresponds to $\left.E\right|_{X_{p(s)}}$, is actually a holomorphic map. $F$.

We shall describe the underlying $C^{\infty}$ fiber bundle $M_{T}^{P}$ over $T$ for the projection

Denote the complement

$$
X_{T}-\bigcup_{i=1}^{n} \operatorname{image}\left(s_{i}\right)
$$

by $X_{T}^{0}$. Also denote the restriction of $f$ to $X_{T}^{0}$ by $f_{0}$; for any $t \in T$, the fiber $f_{0}^{-1}(t)$ will be denoted by $X_{t}^{0}$. Let

$$
\bar{f}: f_{0}^{*} X_{T}^{0} \longrightarrow X_{T}^{0}
$$

be the pull-back, to $X_{T}^{0}$, of the family of punctured Riemann surfaces over $T$. Consider the fundamental groups of the fibers of $\bar{f}$ using the tautological section of $\bar{f}$ as the base points. This family of groups, parametrized by $X_{T}^{0}$, or more precisely the direct image,

$$
\bar{\Pi}=\bar{f}_{*} \pi_{1}
$$

is a locally constant sheaf on $X_{T}^{0}$.

Consider the fiber bundle over $X_{T}^{0}$ given by the space of irreducible representations from fibers of $\bar{\Pi}$ to $U(r)$ such that for any $x \in X_{T}^{0}$, the conjugacy class in the fiber $\bar{\Pi}_{x}$, given by a positively oriented loop around $s_{i} \circ f_{0}(x)$, coincides with the conjugacy 
class $C_{i}$. Denote this fiber bundle over $X_{T}^{0}$ by $Y_{0}$. So local sections of the fiber bundle $Y_{0}$ are simply smoothly varying irreducible representations from fibers of $\bar{\Pi}$ to $U(r)$ satisfying the above holonomy condition.

Now the fiber bundle $Y_{0} \longrightarrow X_{T}^{0}$ has a natural flat connection whose local flat sections are spaces of homomorphisms which are actually locally constant maps from $\bar{\Pi}$, i.e., the homomorphisms do not depend on points of $X_{T}^{0}$.

Let $\bar{Y}$ denote the quotient space for the conjugation action of $U(r)$ on the fiber bundle $Y_{0}$; this quotient is also a fiber bundle over $X_{T}^{0}$. Since the fundamental groups of a manifold with respect to two different base points are naturally isomorphic up to an inner automorphism, the fiber bundle $\bar{Y}$ over $X_{T}^{0}$ descends to a fiber bundle over $T$. This fiber bundle is precisely the underlying $C^{\infty}$ manifold for $M_{T}^{P}$ defined in (3.2).

The flat connection on the fiber bundle $Y_{0}$ induces a $C^{\infty}$ flat connection on the fiber bundle $M_{T}^{P}$. We shall denote this connection by $\hat{\nabla}$.

The following lemma describes the almost complex structure on $M_{T}^{P}$ underlying its complex structure.

Lemma 3.6. For any $t \in T$ and $\rho \in M_{t}^{P}$ let $W \subset T_{\rho}^{\mathbb{R}} M_{T}^{P}$ be the horizontal subspace, of the real tangent space, for the connection $\hat{\nabla}$. Then $W$ is closed under the almost complex structure on $T_{\rho}^{\mathbb{R}} M_{T}^{P}$.

Before actually proving Lemma 3.6 let us first see how this lemma describes the almost complex structure on $M_{T}^{P}$.

The connection $\hat{\nabla}$ decomposes the tangent space $T_{\rho}^{\mathbb{R}} M_{T}^{P}$ as follows:

$$
T_{\rho}^{\mathbb{R}} M_{T}^{P}=T_{\rho}^{\mathbb{R}} M_{t}^{P} \bigoplus W=T_{\rho}^{\mathbb{R}} M_{t}^{P} \bigoplus T_{t}^{\mathbb{R}} T
$$

In Proposition 2.2 and in the proof of Theorem 2.11 we described the complex structure of $M_{t}^{P}$. Now since the map $F$ in (3.2) is holomorphic, Lemma 3.6 implies that the horizontal lift of the almost complex structure on $T_{t}^{\mathbb{R}} T$ is actually the restriction, to the subspace $W$, of the almost complex structure on $T_{\rho}^{\mathbb{R}} M_{T}^{P}$. Thus the almost complex structures on $T_{\rho}^{\mathbb{R}} M_{t}^{P}$ and $T_{t}^{\mathbb{R}} T$ determine the almost complex structure on $T_{\rho}^{\mathbb{R}} M_{T}^{P}$ using decomposition (3.7).

Proof of Lemma 3.6. Take a contractible open subset $U \subseteq T$ containing $t$. So the fundamental group of the space $X_{t}^{0}$, defined in (3.4), maps isomorphically onto $X_{U}^{0}$ using the inclusion of $X_{t}^{0}$ in $X_{U}^{0}$. Using this isomorphism, $\rho$ gives an irreducible flat unitary connection on $X_{U}^{0}$. Taking the Deligne extension along the divisors image $\left(\left.s_{i}\right|_{U}\right)$ we get a parabolic bundle, which we shall denote by $E_{*}$, over $X_{U}$. From the property of $M_{T}^{P}$ described in (3.3) we conclude that the map $\phi_{E_{*}}$ from $U$ to $M_{T}^{P}$, that corresponds to $E_{*}$, is holomorphic. On the other hand, $\phi_{E_{*}}$ is precisely the (unique) horizontal section over $U$, of the fiber bundle $M_{T}^{P}$, which passes through $\rho$. This completes the proof of the lemma.

REMARK 3.8. The projection onto the first factor in the direct sum (3.7) gives a homomorphism

$$
\hat{\nabla}: T^{\mathbb{R}} M_{T}^{P} \longrightarrow T_{\text {rel }}^{\mathbb{R}}
$$

where $T_{\text {rel }}^{\mathbb{R}}:=\operatorname{kernel}(d F)$ is the relative tangent bundle. Lemma 3.6 implies that the above projection $\hat{\nabla}$ is complex linear, i.e., commutes with almost complex structures. However the induced homomorphism of holomorphic tangent bundle, namely

$$
\gamma: T M_{T}^{P} \longrightarrow T_{\text {rel }}
$$


induced by $\hat{\nabla}$, is not a holomorphic homomorphism. This non-holomorphicity of $\gamma$ is a consequence of the fact that the complex structure of the moduli space $M_{t}^{P}$ depends upon the complex structure of the punctured curve $X_{t}^{0}$.

Let

$$
Q_{T}: R_{T}^{P} \longrightarrow T
$$

denote the holomorphic fiber bundle over $T$ whose typical fiber is the space $R\left(X_{0}\right)$ defined in Section 2. So $R_{T}^{P}$ is the fiber bundle over $X_{T}^{0}$ given by the space of equivalence classes of representations from fibers of $\bar{\Pi}$ (defined in (3.5)) to $G L(r)$, such that for any $x \in X_{T}^{0}$, the conjugacy class in the fiber $\bar{\Pi}_{x}$, given by a positively oriented loop around $\left(s_{i} \circ f_{0}\right)(x)$, coincides with the conjugacy class $\bar{C}_{i}$ (defined in Section 2), and moreover the corresponding family of parabolic bundles over marked Riemann surfaces, given by the Deligne extension, are all parabolic stable.

The fiber bundle $R_{T}^{P}$ is holomorphically locally trivial. Moreover, the connection on $R_{T}^{P}$ - defined exactly as $\hat{\nabla}$ on $M_{T}^{P}$ - is actually a holomorphic connection.

Let

$$
q: R_{T}^{P} \longrightarrow M_{T}^{P}
$$

be the obvious projection - which for any $t \in T$ restricts to the projection defined in (2.1).

From Section 2 we know that $q$ is an affine bundle for $\Omega_{\text {rel }}^{1}$, where $\Omega_{\text {rel }}^{1}:=T_{\text {rel }}^{*}$ is the relative cotangent bundle for the projection $F$ defined in (3.2).

The map $\psi$ defined in (2.8) gives a section of $q$, namely

$$
\psi_{T}: M_{T}^{P} \longrightarrow R_{T}^{P}
$$

with $q \circ \psi_{T}=i d$. Just as done in (2.10), we construct the conjugate linear homomorphism for the differential of $\psi_{T}$, namely

$$
\overline{d \psi_{T}}: T M_{T}^{P} \longrightarrow \Omega_{\mathrm{rel}}^{1}
$$

where $\Omega_{\mathrm{rel}}^{1}$, as before, is the relative cotangent bundle for $F$. We shall identify the homomorphism $\overline{d \psi_{T}}$.

Let $\Theta_{T}$ denote the relative 2-form on $R_{T}^{P}$, for the projection $Q_{T}$, which restricts to the form $\Theta$, defined in Section 2, on the fibers of $Q_{T}$. So $\psi_{T}^{*} \Theta_{T}$ is a relative 2 -form on $M_{T}^{P}$.

Let $\gamma$ denote the projection obtained in (3.9). Now

$$
\hat{\Theta}_{T}=\gamma^{*}\left(\psi_{T}^{*} \Theta_{T}\right)
$$

is a 2 -form on $M_{T}^{P}$, with $\psi_{T}^{*} \Theta_{T}$ as the corresponding relative form.

THEOREM 3.12. The homomorphism $\overline{d \psi_{T}}$ is skew-hermitian and it coincides with $\hat{\Theta}_{T} / 2$.

Proof. In view of Theorem 2.11 it is enough to prove that for the subspace $\operatorname{kernel}(\gamma)$, i.e., the horizontal subspace, of $T M_{T}^{P}$ the following holds: (i) $\overline{d \psi_{T}}$ vanishes on $\operatorname{kernel}(\gamma)$; and (ii) the contraction of the form $\hat{\Theta}_{T}$ with any tangent vector in $\operatorname{kernel}(\gamma)$ is zero.

The first statement is a consequence of Lemma 3.6 which ensures that the restriction of the map $\psi_{T}$ to a horizontal leaf, for the flat connection $\hat{\nabla}$ on $M_{T}^{P}$, is actually a holomorphic embedding. 
The second statement is immediate from the definition of $\hat{\Theta}_{T}$, since $\gamma$ vanishes on $\operatorname{kernel}(\gamma)$.

Theorem 3.12 implies that $\hat{\Theta}_{T} / 2$, considered as an element of $H^{1}\left(M_{T}^{P}, \Omega_{\text {rel }}^{1}\right)$, coincides with the cohomology class corresponding to the $\Omega_{\text {rel }}^{1}$-affine bundle $R_{T}^{P}$.

4. Parabolic determinant line bundle over the universal moduli space of parabolic bundles.

4a. The parabolic determinant line. We continue with the notation of Section 2. Henceforth all the parabolic weights will be assumed to be rational numbers.

Let $E_{*}$ be a parabolic vector bundle of rank $r$ over a Riemann surface $X$, with $E$ as the underlying vector bundle, and with $D$ as the set of parabolic points. On $s \in D$

$$
F_{s}^{1}=E_{s} \supset F_{s}^{2} \supset F_{s}^{3} \supset \ldots \supset F_{s}^{l_{s}} \supset F_{s}^{l_{s}+1}=0
$$

is the parabolic flag, and

$$
0 \leq \alpha_{s}^{1}<\alpha_{s}^{2}<\alpha_{s}^{3}<\ldots<\alpha_{s}^{l_{s}}<\alpha_{s}^{l_{s}+1}=1
$$

are the parabolic weights. Assume that

$$
\alpha_{s}^{i}=\frac{m_{s}^{i}}{N}
$$

where $N$ is a fixed positive integer, independent of $s$, and $m_{s}^{i}$ is a nonnegative integer with $0 \leq m_{s}^{i}<N$, and $N$ is the smallest integer satisfying these two conditions. Let $d \in \mathbb{Z}$ be the degree of the underlying vector bundle $E$ for the parabolic bundle $E_{*}$.

Fix a point $x \in X-D$ in the complement of $D$. Following [9] (page 61, Definition 4.8 ) and [19] define the parabolic determinant line to be the following tensor product:

$$
\begin{gathered}
\operatorname{det}\left(E_{*}\right)=\left(\bigwedge^{\mathrm{top}} H^{0}(X, E)\right)^{\otimes-N \cdot \operatorname{rank} E} \bigotimes\left(\bigwedge^{\mathrm{top}} H^{1}(X, E)\right)^{\otimes N \cdot \operatorname{rank} E} \bigotimes \\
\bigotimes_{s \in D, i \in\left[0, l_{s}\right]}\left(\bigwedge^{\mathrm{top}} \frac{F_{s}^{i}}{F_{s}^{i+1}}\right)^{-m_{s}^{i}} \bigotimes\left(\bigwedge^{\mathrm{rank} E} E_{x}\right)^{\otimes\left(N \chi(E)+\sum_{\left.s \in D, i \in\left[0, l_{s}\right] \operatorname{dim}\left(F_{s}^{i} / F_{s}^{i+1}\right) m_{s}^{i}\right)}\right.}
\end{gathered}
$$

where $\chi(E)=\operatorname{dim} H^{0}(X, E)-\operatorname{dim} H^{1}(X, E)$ is the Euler characteristic.

The induced action of an automorphism of $E_{*}$, given by the multiplication by nonzero scalars on $E$, on the line $\operatorname{det}\left(E_{*}\right)$ is the trivial action ([9], page 60).

Assume that the family of Riemann surfaces, given by $f$ in (3.1), is equipped with a section

$$
s: T \longrightarrow X_{T}
$$

such that the image of $s$ does not intersect the image of any $s_{i}$. For this family of Riemann surfaces consider the family of moduli spaces of parabolic bundles, namely $M_{T}^{P}$, defined in Section 3 .

For any point of $M_{T}^{P}$ consider the parabolic determinant line constructed in (4.2). These lines fit together to give a holomorphic line bundle over $M_{T}^{P}$, which we shall call the parabolic determinant line bundle, and it will be denoted by $\mathcal{L}_{T}$; for any $t \in T$, the point $s(t)$ is used as point $x$ in (4.2). We shall briefly explain the construction of 
the line bundle $\mathcal{L}_{T}$. First note that the parabolic determinant line bundle is defined for a family of parabolic vector bundle. Indeed, by [16], [20] the usual determinant line bundle is defined for a family. Now using the parabolic flag for the family the construction of the parabolic determinant line bundle is immediate. Though in general there is no universal parabolic bundle over the fiber product $X_{T} \times_{T} M_{T}^{P}$, we may cover $M_{T}^{P}$ by analytic open sets - alternatively, use open sets in étale topology - such that over each such open set the universal family exists. Construct the parabolic determinant line bundle over these open sets. Now using the earlier mentioned property that the action of $\mathbb{C}^{*}$ is trivial on the determinant lines, these line bundles canonically patch together to give the line bundle $\mathcal{L}_{T}$ over $M_{T}^{P}$. Indeed, any automorphism of a parabolic stable bundle preserving the parabolic flag is a multiplication by a nonzero scalar. So the line bundles over any intersection of two open sets patch satisfying the obvious compatibility condition for intersection of three open sets.

We shall equip $\mathcal{L}_{T}$ with a hermitian metric. Before that we shall first recall the construction of this hermitian metric for the special case of a single Riemann surface, i.e., where $T$ is a point, done in [9].

The moduli space of parabolic bundles, namely $M\left(X_{0}\right)$, defined in Section 2, can be realized as a moduli space of orbifold bundles. This means that there is a finite Galois cover

$$
g: Y \rightarrow X
$$

with Galois group $G$, with $Y$ connected, such that $M\left(X_{0}\right)$ is identified with $M_{G}(Y)$, a moduli of orbifold stable bundle over $Y$. An orbifold bundle is a vector bundle over $Y$ equipped with a lift of the action of $G$; an orbifold bundle $V$ is called stable if for any proper holomorphic subbundle $F$ of $V$, invariant under the action of $G$, the inequality

$$
\frac{\operatorname{deg}(F)}{\operatorname{rank}(F)}<\frac{\operatorname{deg}(V)}{\operatorname{rank}(V)}
$$

is valid. We shall describe the above mentioned identification between parabolic and orbifold bundles in Section 4c. By this identification, the Kähler form $\Theta$ on $M\left(X_{0}\right)$ coincides with $1 / \# G$ times a natural Kähler form on $M_{G}(Y)$ (Lemma 5.1 of [9]); $\# G$ is the order of $G$. The Kähler form on $M_{G}(Y)$ in question is defined by simply copying the definition of the Kähler form $\Theta$, defined in Section 2, for moduli space of usual stable vector bundles. In particular, this form coincides with the pull-back of the Kähler form on the moduli space of (usual) semistable vector bundles using the forgetful map, by forgetting the orbifold structure.

Let $N(\xi) \subseteq M\left(X_{0}\right)$ denote the subvariety consisting of parabolic stable bundles $E_{*}$ with $\bigwedge^{r} E=\xi$, where $\xi$ is a fixed line bundle over $X$. By the above mentioned identification between parabolic and orbifold bundles, $N(\xi)$ gets identified with a moduli space $N_{G}(Y)\left(\subseteq M_{G}(Y)\right)$ of orbifold stable bundles with a fixed determinant.

Using Quillen's construction in [20], a determinant line bundle, denoted by $L^{G}$, over $M_{G}(Y)$, equipped with a hermitian structure, was constructed in Section 3 of [9] where we called it $L^{\pi}$. The fiber of $L^{G}$ over $V \in M_{G}(Y)$ is

$$
\left(\bigwedge^{\mathrm{top}} H^{0}(Y, V)\right)^{\otimes-\mathrm{rank} V} \bigotimes\left(\bigwedge^{\mathrm{top}} H^{1}(Y, V)\right)^{\otimes \mathrm{rank} V} \bigotimes\left(\bigwedge^{\mathrm{rank} V} V_{y}\right)^{\chi(V)}
$$

where $y \in Y$ is a fixed base point; in other words, the definition of $L^{G}$ is same as that for the determinant line bundle over a moduli space of (usual) semistable vector 
bundles. So $L^{G}$ is the pull-back, using the forgetful map mentioned above, of the determinant line bundle over the moduli space of (usual) semistable vector bundles. The curvature of $L^{G}$ coincides with $r$-times the earlier mentioned Kähler form on $M_{G}(Y)$ (Theorem 3.27 of [9]).

The $N \# G$-th tensor power $(N$ as in (4.1)) of the parabolic determinant line bundle over $N(\xi)$ is isomorphic to the restriction of $\left(L^{G}\right)^{\otimes N}$ to $N_{G}(Y)$ (Proposition 4.14 of [9]). Since two choices of such isomorphisms differ by a constant scalar, the metric on the parabolic determinant line bundle induced by this isomorphism is welldefined up to a multiplication by a real positive constant scalar. So the corresponding unitary connection on the parabolic determinant line bundle is uniquely determined. Moreover, the unitary connection on the parabolic determinant line bundle obtained above does not depend upon the choice of the orbifold cover $g$ in (4.3).

The problem of carrying out this project for families of Riemann surfaces breaks into two problems.

The first problem is to have a construction of a "canonical" orbifold cover, satisfying certain properties, for a family of Riemann surfaces. The properties in question include base change property, that is for a pull-back of a family of Riemann surfaces the corresponding orbifold cover should also be the pull-back.

The second problem is to compare the parabolic and the orbifold determinant line bundles. That is to compare, intrinsically, the two lines defined in (4.2) and (4.4) respectively, where $V$ is the orbifold bundle corresponding to the parabolic bundle $E_{*}$.

The second problem has been partially solved in [9] to the extent it is necessary to cover the case of moduli spaces of parabolic bundles with fixed determinant over a fixed Riemann surface. We shall see in (4.19) that the exact form of Proposition 4.14 of [9], where the parabolic and the orbifold determinant line bundles are compared, is not valid for moduli spaces of parabolic bundles, without fixed determinant, on a fixed Riemann surface - a "correction" factor is necessary.

We shall start with the second problem by proving a result (Lemma 4.6) which compares the determinant of a vector bundle with that of its pull-back.

4b. Comparison of determinant of a vector bundle with that of its pullback. Let $E$ be a vector bundle over $X$ of rank $r$. Let $W:=g^{*} E$ be the pull-back bundle over $Y$, where $g$, as in (4.3), is a (possibly ramified) Galois covering. Let $D^{\prime}$ denote the ramification divisor (with multiplicity) on $Y$. So $D^{\prime}$ is the divisor of the natural section of $\left(g^{*} K_{X}\right)^{*} \otimes K_{Y}$.

For any line bundle $L$ over $Y$, define $D^{\prime}(L)$ to be the following line:

$$
D^{\prime}(L):=\bigwedge^{\text {top }} H^{0}\left(X, \frac{L}{L \otimes \mathcal{O}_{X}\left(-D^{\prime}\right)}\right)
$$

Denote the following line by $\mathrm{d}(E)$ :

$$
d(E):=\bigwedge^{\text {top }} H^{0}(Y, E) \bigotimes \bigwedge_{\text {top }}^{1} H^{1}(Y, E)^{*}
$$

Similarly define the line $d(W)$ for $W$.

Lemma 4.6. The two complex one dimensional vector spaces, namely

$$
\left(d(W) \otimes d\left(\mathcal{O}_{Y}\right)^{\otimes-r}\right)^{\otimes 12} \otimes D^{\prime}\left(\bigwedge^{r} W\right)^{\otimes 6} D^{\prime}\left(\mathcal{O}_{X}\right)^{\otimes-6}
$$


and

$$
\left(d(E) \otimes d\left(\mathcal{O}_{X}\right)^{\otimes-r}\right)^{\otimes 12 \# G}
$$

are canonically isomorphic, where \#G is the order of the Galois group $G$, and $\mathcal{O}_{X}$ (respectively $\mathcal{O}_{Y}$ ) is the trivial line bundle over $X$ (respectively $Y$ ).

The word "canonical" above means that for a family of Riemann surfaces as in (3.1), and a Galois covering $\tilde{g}: Y_{T} \longrightarrow X_{T}$ over $T$, and a vector bundle $E_{T}$ over $X_{T}$, the point-wise isomorphisms, over $T$, between two lines, given by Lemma 4.6, fit together to give an isomorphism between the two corresponding line bundles over $T$.

Proof of Lemma 4.6. The key input in the proof is a "Riemann-Roch isomorphism" established by Deligne in [12] (Théorème 9.9, page 170).

The first step in the proof will be to establish the following identification in the notation of Section 9 of [12]:

$$
I_{X} C^{2}(E)^{\otimes \# G}=I_{Y} C^{2}(W)
$$

Since the parameter space $S$ (in [12]) is a single point here, we omit "S" it in the notation of $I_{X / S} C^{2}$ in [12].

The above identification (4.7) is a consequence of (2.12) of [4] (page 155) and 9.1.4 of [12] (page 155). The isomorphism (2.12) of [4] is easily seen to be valid for ramified coverings. Though the covering $p$ in $((2.12),[4])$ is unramified - the same proof goes through; the assumption on unramifiedness is used only in the proof of isometricity of (2.12).

To prove (4.7) take a flag of subbundles of $E$, namely

$$
E=E_{r} \supset E_{r-1} \supset E_{r-2} \supset \ldots \supset E_{1} \supset E_{0}=0 \text {, }
$$

such that all subsequent quotients are line bundles, as done in 9.5 (page 159) of [12]. The pull-back of this flag gives a flag of subbundles of $W$. Using (9.1.4) of [12] we express $I_{X} C^{2}(E)$ and $I_{Y} C^{2}(W)$ as follows:

$$
I_{X} C^{2}(E)=\bigotimes_{i=1}^{r-1}\left\langle\bigwedge^{i} E_{i}, E_{i+1} / E_{i}\right\rangle \text { and } I_{Y} C^{2}(W)=\bigotimes_{i=1}^{r-1}\left\langle\bigwedge^{i} g^{*} E_{i}, g^{*}\left(E_{i+1} / E_{i}\right)\right\rangle
$$

Now the isomorphism (2.12) in [4], namely

$$
\left\langle\bigwedge^{i} E_{i}, E_{i+1} / E_{i}\right\rangle^{\otimes \# G}=\left\langle\bigwedge^{i} g^{*} E_{i}, g^{*}\left(E_{i+1} / E_{i}\right)\right\rangle
$$

completes the proof of (4.7).

As the next step we shall deduce the following (canonical) equality:

$$
\left(d(E) \otimes d\left(\mathcal{O}_{X}\right)^{\otimes-r}\right)^{\otimes 12}=\left\langle\bigwedge^{r} E, \bigwedge^{r} E \otimes T_{X}\right\rangle^{\otimes 6} \otimes I_{X} C^{2}(E)^{\otimes-12}
$$

We first observe that by Théorème 9.9 (iii), page 170, of [12], the following equality is valid:

$$
d\left(\mathcal{O}_{X}^{\oplus r}\right)^{\otimes 12}=\left\langle K_{X}, K_{X}\right\rangle^{\otimes r}
$$


Indeed, that the other two terms in the right hand side of Théorème 9.9(iii) are trivial for $d\left(\bigoplus_{r} \mathcal{O}_{X}\right)^{\otimes 12}$ follows from the combination of the following identifications (Proposition 5(a), page 367, [2]):

$$
\left\langle L, \mathcal{O}_{X}\right\rangle=\mathbb{C} \quad \text { and } \quad\left\langle L, L_{1} \otimes L_{2}\right\rangle=\left\langle L, L_{1}\right\rangle \otimes\left\langle L, L_{2}\right\rangle,
$$

where $L, L_{1}$ and $L_{2}$ are line bundles over $X$, with 9.1.4 of [12] which ensures that $I_{X} C^{2}\left(\mathcal{O}_{X}^{\oplus r}\right)=\mathbb{C}$.

Now applying Théorème 9.9(iii) of [12] to $E$, and noting that $d\left(\mathcal{O}_{X}^{\oplus r}\right)=d\left(\mathcal{O}_{X}\right)^{\otimes r}$, the identification in (4.9) is immediate.

Similarly we have the following equality

$$
\left(d(W) \otimes d\left(\mathcal{O}_{Y}\right)^{\otimes-r}\right)^{\otimes 12}=\left\langle\bigwedge^{r} W, \bigwedge^{r} W \otimes T_{Y}\right\rangle^{\otimes 6} \otimes I_{Y} C^{2}(W)^{\otimes-12}
$$

In view of (4.7), comparing the right-hand-sides of (4.9) and (4.11) we conclude that in order to complete the proof of the lemma it is enough to show that

$$
\left\langle\bigwedge^{r} W, \bigwedge^{r} W \otimes T_{Y}\right\rangle^{\otimes 6} \otimes D^{\prime}\left(\bigwedge^{r} W\right)^{\otimes 6} D^{\prime}\left(\mathcal{O}_{X}\right)^{\otimes-6}=\left\langle\bigwedge^{r} E, \bigwedge^{r} E \otimes T_{X}\right\rangle^{\otimes 6 \# G}
$$

By the distributive property in (4.10), we have

$$
\left\langle\bigwedge^{r} E, \bigwedge^{r} E \otimes T_{X}\right\rangle=\left\langle\bigwedge^{r} E, \bigwedge^{r} E\right\rangle \otimes\left\langle\bigwedge^{r} E, T_{X}\right\rangle
$$

Thus, since $W=g^{*} E$, using the equality (2.12) of [4] (which is same as (4.8) above) we conclude that (4.12) is a consequence of the following:

$$
\left\langle\bigwedge^{r} W, T_{Y}\right\rangle \otimes D^{\prime}\left(\bigwedge^{r} W\right) \otimes D^{\prime}\left(\mathcal{O}_{X}\right)^{\otimes-1}=\left\langle\bigwedge^{r} W, g^{*} T_{X}\right\rangle
$$

Applying (4.10) again, the above equality is equivalent to

$$
D^{\prime}\left(\bigwedge^{r} W\right) \otimes D^{\prime}\left(\mathcal{O}_{X}\right)^{\otimes-1}=\left\langle\bigwedge^{r} W, K_{Y} \otimes g^{*} T_{X}\right\rangle
$$

By definition, $\mathcal{O}_{X}\left(D^{\prime}\right)=K_{Y} \otimes g^{*} T_{X}$. So the above equality is a special case of Proposition 5(c) (page 367) of [2]. (In this Proposition 5(c) of [2] replace $L$ by $\bigwedge^{\top} W$ and $D$ by $D^{\prime}$.) This completes the proof of the lemma.

In the next subsection we shall use Lemma 4.6 to solve the second of the two problems mentioned at the end of Section $4 \mathrm{a}$.

4c. Comparison between parabolic and orbifold determinant lines. In this subsection we shall first describe the correspondence between parabolic and orbifold bundles mentioned earlier; this will be used in the next step of our computation for comparison between parabolic and orbifold determinant lines. A description of this identification can be found in [9], where orbifold bundles are called $\pi$-bundles following [21]. The details of the identification of parabolic bundles and orbifold bundles is incomplete in [9], but the complete details can be found in [5], where the base $X$ is allowed to have arbitrary dimension.

Given a parabolic data for a Riemann surface $X$ with $D$ as the parabolic points, there is a finite Galois cover $g: Y \longrightarrow X$, as in (4.3), such that $g$ is ramified over all points of $D$ (there may be other ramification points also), such that multiplicity of $g$ 
over any $s \in D$ is a multiple of $N$, where $N$ as in (4.1). A proof of the existence of such a cover can be found in Theorem 1.2.15, page 29, of [18].

An orbifold bundle on $Y$ is a vector bundle over $Y$ equipped with a lift of the action of the Galois group.

Let $E_{*}$ be a parabolic bundle over $X$ of rank $r$ with parabolic structure as given in the beginning of Section $4 a$.

Let the multiplicity of $g$ at $s \in D$ be $k_{s} N$; in other words, $g^{*} s=k_{s} N\left(g^{*} s\right)_{\text {red }}$.

For $s \in D$, denote the reduced divisor $\left(g^{*} s\right)_{\text {red }}$ by $\tilde{s}$. Consider the vector space

$$
Q_{s}^{j}:=\frac{E_{s}}{F_{s}^{j}} \bigotimes \mathcal{O}_{X}(s)_{s}=\frac{E_{s}}{F_{s}^{j}} \bigotimes T_{s} X
$$

as a torsion sheaf on $X$ supported on $s$. Let

$$
\left.g^{*} Q_{s}^{j} \longrightarrow g^{*} Q_{s}^{j}\right|_{k_{s}\left(N-m_{s}^{j-1}\right) \tilde{s}} \longrightarrow 0
$$

be the restriction of $g^{*} Q_{s}^{j}$, which is supported on $g^{*} s$, to the (non-reduced) divisor $k_{s}\left(N-m_{s}^{j-1}\right) \tilde{s}$.

The pull-back bundle $g^{*}\left(E \otimes \mathcal{O}_{X}(D)\right)$ has a natural projection onto $g^{*} Q_{s}^{j}$ given by restriction. For any $\tilde{s} \in g^{-1}(s)$, composing this with the homomorphism in (4.13) we get a projection

$$
\left.0 \longrightarrow U_{\tilde{s}}^{j} \longrightarrow g^{*}\left(E \bigotimes \mathcal{O}_{X}(D)\right) \longrightarrow\left(g^{*} Q_{s}^{j}\right)\right|_{k_{s}\left(N-m_{s}^{j-1}\right) \tilde{s}} \longrightarrow 0
$$

where $U_{\tilde{s}}^{j}$ is defined to be the kernel of this projection.

Finally, define

$$
V:=\bigcap_{s \in g^{-1}(D), j=1}^{j=l_{g(s)}} U_{\tilde{s}}^{j}
$$

to be the intersection of all subsheaves $U_{\tilde{s}}^{j}$ of $g^{*}\left(E \otimes \mathcal{O}_{X}(D)\right)$.

There is a natural action of the Galois group $G$ on $f^{*}\left(E \otimes \mathcal{O}_{X}(D)\right)$, since it is a pull-back from $X$, which commutes with the Galois action of $G$ on $Y$. The subsheaf $V$ is left invariant by this action, and hence $V$ gets an induced orbifold bundle structure.

In the correspondence between parabolic bundles and orbifold bundles, the orbifold bundle $V$ over $Y$, constructed above, corresponds to the parabolic bundle $E_{*}$. It is easy to check that $E_{*}$ is parabolic stable if and only if $V$ is orbifold stable [5].

For $s \in g^{-1}(S)$, define

$$
G_{\tilde{s}}^{j}:=\left.g^{*}\left(F_{g(s)}^{j} / F_{g(s)}^{j+1}\right)\right|_{\tilde{s}}
$$

to be the torsion sheaf supported (scheme theoretically) on the reduced divisor $\tilde{s}$.

Using the natural exact sequence

$$
0 \longrightarrow \mathcal{O}_{Y}((c-1) \tilde{s}) \longrightarrow \mathcal{O}_{Y}(c \tilde{s}) \longrightarrow T_{\tilde{s}}^{\otimes c} Y \longrightarrow 0
$$

we conclude the following $(d(-)$ defined in (4.5)):

$$
d(V)=d\left(g^{*} E\right) \bigotimes\left(\bigotimes_{s \in g^{-1}(D), j=1, i=1}^{j=l_{g(s)}, i=k_{g(s)} m_{g(s)}^{j}} \bigwedge^{\mathrm{top}}\left(G_{\tilde{s}}^{j} \otimes T_{\tilde{s}}^{\otimes i} Y\right)\right)
$$


To prove (4.16), in conjunction with the above construction of $V$ we need to use the fact that if the quotient of an injective homomorphisms of coherent sheaves on $Y$, say

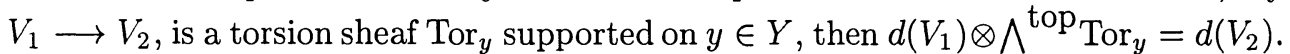
An equality similar to (4.16) can be found in (3.15) of [5].

Combining (4.16) with Lemma 4.6 we get the following:

THEOREM 4.17. There is a canonical isomorphism between the following two lines:

$$
\begin{gathered}
\left(d(V) \bigotimes d\left(\mathcal{O}_{Y}\right)^{\otimes-r}\right)^{\otimes 12} \bigotimes D^{\prime}\left(\bigwedge^{r} g^{*} E\right)^{\otimes 6} D^{\prime}\left(\mathcal{O}_{X}\right)^{\otimes-6} \\
=\left(d(E) \bigotimes d\left(\mathcal{O}_{X}\right)^{\otimes-r}\right)^{\otimes 12 \# G} \bigotimes\left(\bigotimes_{s \in g^{-1}(D), j=1, i=1}^{j=l_{g(s)}, i=k_{g(s)} m_{g(s)}^{j}} \bigwedge^{\text {top }}\left(G_{\bar{s}}^{j} \otimes T_{\bar{s}}^{\otimes i} Y\right)\right)^{\otimes 12}
\end{gathered}
$$

Let us first see how Theorem 4.17 immediately implies the main result [Proposition 4.14] of [9] mentioned earlier.

The correspondence between parabolic bundles and orbifold bundles gives an identification of a moduli of parabolic stable bundles over $X$ with a moduli of orbifold stable bundles over $Y$.

If we have a fixed covering between two fixed Riemann surfaces (not in a family) then we may choose and fix trivializations of $d\left(\mathcal{O}_{Y}\right), d\left(\mathcal{O}_{X}\right), T_{\tilde{s}} Y$ and $D^{\prime}\left(\mathcal{O}_{X}\right)$ over the moduli space of parabolic (or equivalently orbifold) stable bundles. If, furthermore, we restrict ourselves to a moduli of parabolic bundles with a fixed determinant, then $D^{\prime}\left(\bigwedge^{r} g^{*} E\right)$ is also trivializable over the moduli space. Thus, in that case, Theorem 4.17 induces an isomorphism between $12 N$-th tensor power of the orbifold determinant line bundle, defined by (4.4), over moduli space of orbifold stable bundles with a fixed determinant, and the $12 \# G$-th tensor power of the parabolic determinant line bundle, defined using (4.2). This isomorphism depends upon the choices of trivializations of various lines mentioned above, but any two such isomorphisms clearly differ by a constant scalar. (Take the point $x$ in (4.2) such that $g(y)=x$; so $\bigwedge^{r} E_{x}=\bigwedge^{r} V_{y}$.) This establishes Proposition 4.14 of [9].

Let $M\left(X_{0}\right)$ denote the moduli space of parabolic stable bundles (without fixing the determinant) over $X$. Let $M_{G}(Y)$ denote the corresponding moduli space of orbifold stable bundles over $Y$. Let $L_{P}$ (respectively $L_{G}$ ) denote the determinant line bundle, defined using (4.2) (respectively (4.4)), over $M\left(X_{0}\right)$ (respectively $M_{G}(Y)$ ). Take the point $y \in Y$ such that $g(y)=x$.

For any $E \in M\left(X_{0}\right)$, define the line $L(E, g, N)$ by

$$
L(E, g, N)
$$

$$
\begin{aligned}
& :=D^{\prime}\left(\bigwedge^{r} g^{*} E\right)^{\otimes-6 N} \bigotimes\left(\bigwedge^{r} E_{x}\right)^{12\left(-N \chi(V)+N \# G \chi(E)+\# G \sum_{s \in D, i \in\left[0, l_{s}\right]} \operatorname{dim}\left(F_{s}^{i} / F_{s}^{i+1}\right) m_{s}^{i}\right)} \\
& =D^{\prime}\left(\bigwedge^{r} g^{*} E\right)^{\otimes-6 N} \bigotimes\left(\bigwedge^{r} E_{x}\right)^{12 N\left(-\chi(V)+\# G \chi(E)+\# G \sum_{s \in D, i \in\left[0, l_{s}\right]} \operatorname{dim}\left(F_{s}^{i} / F_{s}^{i+1}\right) \alpha_{s}^{i}\right)}
\end{aligned}
$$

Let $L(g, N)$ be the natural line bundle over $M\left(X_{0}\right)$ whose fiber over $E \in M\left(X_{0}\right)$ is the line $L(E, g, N)$ defined in (4.18). 
Theorem 4.7 implies that

$$
L_{G}^{\otimes 12 N} \bigotimes L(g, N)=L_{P}^{\otimes 12 \# G}
$$

over $M\left(X_{0}\right)=M_{G}(Y)$.

For the special case of $r=1$ and $\# D=1$ it is easy to check that the line bundle $L(g, N)$ in (4.19) is, in general, a nontrivial line bundle over the Jacobian of $X$.

Let $J(X)$ denote the Jacobian of $X$ consisting of isomorphism classes of topologically trivial line bundles over $X$. It is easy enough to check that the line bundle $L(g, N)$ over $M\left(X_{0}\right)$ is the pull-back of a topologically trivial line bundle over $J(X)$ using the projection

$$
p r: M\left(X_{0}\right) \longrightarrow J(X)
$$

defined by $E \longmapsto \mathcal{O}_{X}(-\operatorname{deg}(E) x) \otimes \bigwedge^{r} E$. The above statement follows from the fact that $\operatorname{deg}\left(D^{\prime}\right)=2\left(-\chi(V)+\# G \chi(E)+\# G \sum_{s \in D, i \in\left[0, l_{s}\right]} \operatorname{dim}\left(F_{s}^{i} / F_{s}^{i+1}\right) \alpha_{s}^{i}\right)$. Let $\bar{L}(g, N)$ denote the line bundle over $J(X)$ satisfying the condition $\operatorname{pr}^{*} \bar{L}(g, N)=L(g, N)$.

From Theorem 3.27 of [9] (page 55) we have a natural hermitian connection on $L_{G}$ whose curvature is the natural Kähler form on $M_{G}(Y)$. Also, the Kähler form $\Theta$ on $M\left(X_{0}\right)$ coincides with $1 / \# G$ times a natural Kähler form on $M_{G}(Y)$ (Lemma 5.1 of [9]). The line bundle $\bar{L}(g, N)$ over $J(X)$, being topologically trivial, has a natural flat unitary connection.

Combining these observations the identification (4.19) implies the following theorem:

THEOREM 4.20. The line bundle $L_{P}$ over $M\left(X_{0}\right)$ has a natural hermitian connection with curvature $\mathrm{Nr} \Theta$.

The hermitian connection on $L_{P}$ obtained in Theorem 4.20 does not depend upon the choice of the covering $g$.

4d. Coverings for families of Riemann surfaces. In this subsection we solve the first one of the two problems mentioned at the end of Section 4a. More precisely, we shall construct compatible orbifold covers for families of Riemann surfaces (given a parabolic data); the compatibility, in question, is with respect to base change for families.

Let $X$ be a compact connected oriented real two manifold. Fix a finite subset $D:=\left\{p_{1}, \ldots, p_{n}\right\} \subset X$. Assume that $(X, D) \neq\left(\mathbb{C P}^{1},\{\right.$ point $\left.\}\right)$. In other words, either $\operatorname{genus}(X) \geq 1$ or $\# D \geq 2$.

Take $x \in X-D$, and set $D^{\prime}=D \cup x$. The following lemma we be used later in this subsection.

Lemma 4.21. For any integer $N \geq 2$, there is a finite ramified Galois cover

$$
g: Y \longrightarrow X
$$

with $Y$ connected, which is unramified outside $D$, and the multiplicity of $g$ over any point of $D$ is a multiple of $N$. Moreover, for any diffeomorphism, say $f$, of $X$ fixing $D^{\prime}$ (point-wise), there is diffeomorphism, denoted by $\bar{f}$, of $Y$, such that the following equality of maps is valid:

$$
\bar{f} \circ g=g \circ f
$$


Proof. By Theorem 1.2.15 (Bundgaard-Nielson-Fox Theorem), page 29, of [18], there is a finite Galois cover

$$
h: Z \longrightarrow X
$$

satisfying the condition that $h$ is unramified over $X-D$ and the multiplicity of $h$ over any point of $D$ is a multiple of $N$.

Take the point $x \in X-D$ and take a point $z_{0} \in h^{-1}(x)$ as base points for defining fundamental groups of $X-D$ and $Z-h^{-1}(D)$ respectively. We first note that using the homotopy lifting property, the proof of the lemma will be completed once we are able to show the following: there is a subgroup $\Gamma \subseteq \pi_{1}\left(Z-h^{-1}(D)\right)$ of finite index such that the image of the composition

$$
\Gamma \longrightarrow \pi_{1}\left(Z-h^{-1}(D)\right) \longrightarrow \pi_{1}(X-D)
$$

is left invariant by any automorphism of $\pi_{1}(X-D)$. Indeed, in that case the covering of $X-D$ given by $\Gamma$, which extends as a ramified cover of $X$, satisfies all the required conditions.

Lemma 3.2 of [8] ensures the existence of such a subgroup $\Gamma$ of $\pi_{1}\left(Z-h^{-1}(D)\right)$. However, to be somewhat self-contained, we shall give the details of the proof. Consider the left multiplication action of $\pi_{1}(X-D)$ on the right coset space

$$
S_{r}=\pi_{1}(X-D) / \pi_{1}\left(Z-h^{-1}(D)\right) .
$$

This gives a homomorphism from $\pi_{1}(X-D)$ to $P\left(S_{c}\right)$, the group of permutations of the finite set $S_{c}$. Let $\kappa$ denote this element of $\operatorname{Hom}\left(\pi_{1}(X-D), P\left(S_{c}\right)\right)$. Now define

$$
\Gamma:=\bigcap_{\gamma \in \operatorname{Hom}\left(\pi_{1}(X-D), P\left(S_{c}\right)\right)} \operatorname{kernel}(\gamma)
$$

It is easy to see that $\Gamma$ is contained in $\pi_{1}\left(Z-h^{-1}(D)\right)$. Indeed, for any $g \in \pi_{1}(X-D)-$ $\pi_{1}\left(Z-h^{-1}(D)\right)$, the action of $\kappa(g)$ on $S_{c}$ is actually nontrivial. Since Hom $\left(\pi_{1}(X-\right.$ $\left.D), P\left(S_{c}\right)\right)$ is a finite group, $\Gamma$ is of finite index in $\pi_{1}\left(Z-h^{-1}(D)\right)$. This completes the proof of the lemma.

Let $\operatorname{Diff}^{+}\left(X, D^{\prime}\right)$ denote the group of orientation preserving diffeomorphisms of $X$ fixing $D^{\prime}$ point-wise.

The Galois group of $g$ is denoted by $G$. Let $\operatorname{Diff}^{+}(Y, G)$ denote the group of all orientation preserving diffeomorphisms of $Y$ which commute with the action of $G$.

Fix a point $y \in g^{-1}(x)$, where $g$ as in Lemma 4.21. Now the diffeomorphism $\bar{f}$ in Lemma 4.21 can be uniquely fixed by imposing the condition that $\bar{f}(y)=y$.

So Lemma 4.21 gives a natural homomorphism

$$
\hat{g}: \operatorname{Diff}^{+}\left(X, D^{\prime}\right) \longrightarrow \operatorname{Diff}^{+}(Y, G)
$$

Suppose we are given a parabolic data, i.e., parabolic weights and their multiplicities. Fix once and for a covering $g$ as in Lemma 4.21 where $N$ as in (4.1).

Let $f$ be a family of Riemann surfaces (as in (3.1)) with $n+1$ marked points, given by $n+1$ disjoint sections, namely $\left\{\left\{s_{i}\right\}_{1 \leq i \leq n}, s\right\}$. Points gives by sections $s_{i}$ are parabolic points, and $s$ will be used to define the parabolic determinant line bundle $\mathcal{L}_{T}$ constructed in Section 4a. 
The family $f$ gives a principal $\operatorname{Diff}^{+}\left(X, D^{\prime}\right)$ bundle over $T$, denoted by $P(f)$, whose fiber $P(f)_{t}$, over $t \in T$, is the space of all orientation preserving diffeomorphisms from $X$ to $X_{t}$ mapping $p_{i}$ and $x$ to $s_{i}(t)$ and $s(t)$ respectively.

Let $P(\hat{g})$ be the principal $\operatorname{Diff}^{+}(Y, G)$-bundle over $T$ obtained from $P(f)$ by extending its structure group using the homomorphism $\hat{g}$ in (4.22).

Let $Y_{T} \longrightarrow T$ be the fiber bundle associated to $P(\hat{g})$ for the obvious action of $\operatorname{Diff}^{+}(Y, G)$ on $Y$.

The covering map $g$ induces a Galois covering map

$$
g_{T}: Y_{T} \longrightarrow X_{T}
$$

with $G$ as the Galois group. This follows from the fact that elements of $\operatorname{Diff}^{+}(Y, G)$ commute with the action of $G$ on $Y$. The complex structure on $X_{T}$ induces a complex structure on $Y_{T}$ such that $g_{T}$ (in (4.23)) is a holomorphic map.

The Galois covering $g_{T}$, which depends only on the choice of $g$ in Lemma 4.21, is the canonical orbifold covering of $X_{T}$. Clearly this covering commutes with base change.

4e. Hermitian connection on parabolic determinant line bundle. Let

$$
\Phi: N_{T}^{P} \longrightarrow T
$$

denote the family of parabolic stable bundles with fixed determinant for the family $f$ (as in Section 4d) of Riemann surfaces; for $t \in T, N_{t}^{P}$ is the moduli space of parabolic stable bundles, $E_{*}$, with the given parabolic data and $\bigwedge^{r} E=\mathcal{O}_{X_{t}}(\operatorname{deg}(E) s(t))$.

Let $N_{T}^{G}$ denote the corresponding family of orbifold stable bundles of fixed determinant for the family $Y_{T}$ constructed in (4.23).

Let $L_{P}^{T}$ (respectively $L_{G}^{T}$ ) denote the determinant line bundle, defined using (4.2) (respectively $(4.4)$ ), over $N_{T}^{P}$ (respectively $N_{T}^{G}$ ). Theorem 4.17 implies that the line bundle

$$
\left(L_{P}^{T}\right)^{\otimes 12 \# G} \bigotimes\left(L_{G}^{T}\right)^{\otimes-12 N}
$$

on $N_{T}^{P}=N_{T}^{G}$ is the pull-back of a line bundle over $T$. Denote by $L(T)$ the line bundle over $T$ such that $\Phi^{*} L(T)$ ( $\Phi$ as in (4.24)) is the line bundle in (4.25).

Associating $L(T)$ to the family $f$ of $(n+1)$-pointed Riemann surface, we get a line bundle over the moduli functor (or moduli stack) of $(n+1)$-pointed Riemann surfaces. (See $\S 1$ of [1] for the definition of a line bundle over the moduli functor.) That the association of $L(T)$ to the family $f$ gives a line bundle over the moduli functor is a consequence of following two facts: (1) the covering $g_{T}$ in (4.23) is compatible with base change for the family $f$. In other words, given a holomorphic map $i: S \longrightarrow T$, the orbifold covering for the pull-back family $i^{*} f$ is precisely the pull-back of the family $Y_{T} \longrightarrow T$ to $S$. (2) the identification of the two complex lines in Theorem 4.17 is canonical.

Assume that genus $\left(X_{t}\right) \geq 3$. Now from the second part of Theorem 2, page 163, of [1] we know that $L(T)$ is a linear combination of the Hodge bundle $\left(=\bigwedge^{\text {top }} f_{*} K_{\text {rel }}\right)$ and the line bundles over $T$ given by the relative tangent lines (for the family $X_{T}$ ) at the marked points. Using the Poincaré metric on the fibers of $X_{T}$, all these line bundles have natural hermitian structures.

Thus we have a natural hermitian structure on $L(T)$. Also, $L_{G}^{T}$ has a natural hermitian structure constructed in [9] using a theorem of Quillen [20]. Thus $L_{P}^{T}$ gets equipped with a natural hermitian structure. 
Consider the relative $(1,1)$-form $\hat{\Theta}_{T}$ considered in Theorem 3.12. Using the projection $\gamma$ in (3.9), the relative form $\hat{\Theta}_{T}$ gives a $(1,1)$-form on $N_{T}^{P}$, which we shall also denote by $\hat{\Theta}_{T}$.

From Theorem 5.4 of [6] we know that the curvature of the hermitian connection on $L_{G}^{T}$ is of the form:

$$
a \hat{\Theta}_{T}+b \Phi^{*} \omega_{W P}
$$

where $a$ and $b$ are purely imaginary numbers and $\omega_{W P}$ is the Weil-Petersson form on $T$ for the family $f$ considered forgetting the marked points.

Thus the curvature of the hermitian connection on $L_{T}^{P}$ is of the form

$$
c \hat{\Theta}_{T}+\Phi^{*} \omega
$$

where $c$ is a purely imaginary number and $\omega$ is a $(1,1)$-form on $T$.

4f. Concluding Remarks. It is known that the moduli space of orbifold semistable vector bundles over a Riemann surface is an irreducible projective variety, and the moduli space of orbifold stable bundles is an open subset of it with the complement being of sufficiently high codimension. Using this it can be deduced that the two Hermitian metrics on the determinant line bundle $L_{G}^{T}$ (defined in Section 4e), corresponding to two different choices of the relative Kähler metrics on the relative tangent bundle, differ by $\exp (f)$, where $f$ is a real valued harmonic function on the parameter space $T$. Indeed, the earlier remark implies that any harmonic function on $N_{T}^{G}$ descends to $T$. In a very general context, the variation of the Quillen metric with respect to the metric in the relative tangent bundle is given by [3, Theorem 0.2]. So, in principle, using this theorem the above function $f$ should be expressible in terms of the choices of the metrics. But we have not been able to work it out.

A similar remark is valid on the dependence of the Hermitian metric of the parabolic determinant line bundle $L_{P}^{T}$ on the choice of the Galois covering.

It will be interesting to be able to construct the Hermitian connection on the parabolic determinant line bundle $L^{P}$, over the moduli space of parabolic stable bundles over a Riemann surface, without using orbifold bundles. To construct a natural Hermitian structure on $L_{P}^{T}$, without using orbifold bundles, would involve generalizing Théorème 11.4 of [12] to the parabolic context. (The constant $a(X)$ in Théorème 11.4 of [12] has been computed in [14].)

\section{REFERENCES}

[1] E. Arbarello and M. Cornalba, The Picard group of the moduli spaces of curves, Topology 26 (1987), 153-171.

[2] A. Beilinson And YU. Manin, The Mumford form and the Polyakov measure in string theory, Comm. Math. Phy. 107 (1986), 359-376.

[3] J.-M. Bismut, H. Gillet AND C. Soulé, Analytic torsion and holomorphic determinant bundles I. Bott-Chern forms and analytic torsion, Comm. Math. Phy. 115 (1988), 49-78.

[4] I. Biswas, S. NAG AND D.P. Sullivan, Determinant bundles, Quillen metrics and Mumford isomorphisms over the universal commensurability Teichmüller space, Acta Math. 176 (1996), 145-169.

[5] I. BISWAS, Parabolic bundles as orbifold bundles, Duke Math. Jour. 88 (1997), 305-325.

[6] I. BiswAs, Determinant bundle over the universal moduli space of vector bundles over the Teichmüller space, Annales Inst. Fourier 47 (1997), 885-914.

[7] I. Biswas AND K. Guruprasad, Principal bundles on open surfaces and invariant functions on Lie groups, Int. Jour. Math. 4 (1993), 535-544. 
[8] I. BISWAS AND S. NAG, Weil-Petersson geometry and determinant bundles on inductive limits of moduli spaces, Lipa's Legacy (Ed. J. Dodziuk and L. Keen), Contemp. Math. 211 (1997), 51-80.

[9] I. Biswas AND N. Raghavendra, Determinants of parabolic bundles on Riemann surfaces, Proc. Ind. Acad. Sci. (Math. Sci.) 103 (1993), 41-71.

[10] I. Biswas AND S. Ramanan, An infinitesimal study of the moduli of Hitchin pairs, Jour. of the Lond. Math. Soc. 49 (1994), 219-231.

[11] P. Deligne, Equations differentielles à points singuliers ŕeguliers, Lecture Notes in Mathematics 163, Springer-Verlag, Heidelberg (1970).

[12] P. Deligne, Le déterminant de la cohomologie, Contemp. Math. 67 (1987), 93-177.

[13] J-M. Drezet and M.S. Narasimhan, Groupes de Picard des variétés de modules de fibrés semistable sur les courbes algébriques, Inventiones Math. 97 (1989), 53-94.

[14] H. Gillet AND C. Soulé, An arithmetic Riemann-Roch theorem, Inv. Math. 110 (1992), 473-543.

[15] N. KaTZ, An overview of Deligne's work on Hilbert's twenty-first problem, Mathematical developments arising from Hilbert Problems, Proc. of Symp. in Pure Math. 28, part 2. (1974), 537-557.

[16] F. KNudsen And D. Mumford, The projectivity of the moduli space of stable curves I. Preliminaries on "det" and "div", Math. Scand. 39 (1976), 19-55.

[17] V. MeHTA AND C. SEShAdRI, Moduli of vector bundles with parabolic structures, Math. Ann. 248 (1980), 205-239.

[18] M. NAMBA, Branched coverings and algebraic functions, Pitman research notes in math. series; 161. Longman Sci. Tech. (1987).

[19] : M.S. NARASImHAN AND T. RAmAdas, Factorisations of generalized theta function, I. Inv. Math. 114 (1993), 565-623.

[20] D. Quillen, Determinants of Cauchy-Riemann operators over a Riemann surface, Funct. An. Appl. 19 (1985), 31-34.

[21] C.S. SEShADRI, Moduli of $\pi$-vector bundles over an algebraic curve, Questions on algebraic varieties. C.I.M.E. III Ciclo, Varenna (1970), 141-260.

[22] C. Simpson, Harmonic bundles on noncompact curves, Jour. of the Amer. Math. Soc. 3 (1990), 713-770.

[23] K. Timmerscheidt, Hodge decomposition for unitary local systems, Appendix D to: H. Esnault and E. Viehweg, Logarithmic de Rham complexes and vanishing theorems. Inv. Math. 86 (1986), 161-194.

[24] K. Timmerscheid , Mixed Hodge theory for unitary local systems, Jour. Reine Angew. Math. 379 (1987), 152-171. 